\title{
ON SETS EVERY HOMEOMORPHIC IMAGE OF WHICH HAS THE BAIRE PROPERTY
}

\author{
JOHN C. MORGAN II
}

\begin{abstract}
It is shown that the class of sets every homeomorphic image of which has the Baire property does not coincide with the class of sets which have the Baire property in the restricted sense.
\end{abstract}

In [2] a method has been given for unifying, in the setting of complete, separable metric spaces, the topological notions of the Baire property in the restricted sense and of a set being always of the first category with the corresponding measure-theoretic notions of absolute measurability and of a set being absolutely of measure zero, respectively. In the case of linear sets, the absolutely measurable sets, investigated by Marczewski in [4], coincide with those sets every homeomorphic image of which is Lebesgue measurable. This latter class of sets, called "perfectly measurable sets" by Urysohn, was studied by Lavrentiev in [1].

The Baire property (in the wide sense) being the appropriate topological analogue of Lebesgue measurability, one might expect, in view of the known analogies, that the sets every homeomorphic image of which has the Baire property (in the wide sense) would coincide with the sets which have the Baire property in the restricted sense. Although the former class of sets necessarily contains the latter class, which is invariant under homeomorphisms, we shall show that they do not coincide.

For the convenience of the reader we recall some pertinent definitions. A set $S$ in a topological space $X$ has the Baire property relative to $X$ if every nonempty set $G$ open in $X$ contains a nonempty set $H$ open in $X$ such that either $H \cap S$ or $H \cap(X-S)$ is of the first category in $X$. A set $S \subset X$ has the Baire property in the restricted sense if, for every perfect set $P \subset X$, the set $S \cap P$ has the Baire property relative to $P$ endowed with the relativized topology.

All sets considered below are assumed to be subsets of the real line, which we shall denote by $X$.

THEOREM 1. There exists a linear set every homeomorphic image of which has the Baire property, but which does not have the Baire property in the restricted sense.

Presented to the Society, October 21, 1978; received by the editors September 5, 1978.

AMS (MOS) rubject classifications (1970). Primary 54A05.

Key words and phrases. Baire category, Baire property, scattered set, homeomorphism, analogies between category and measure. 
Proof. Let $K$ denote the Cantor set and let $A$ be a totally imperfect subset of $K$, consisting only of irrational points, such that $K-A$ is also totally imperfect. The set $S=X-A$ does not have the Baire property relative to $K$ and hence does not have the Baire property in the restricted sense. However, every homeomorphic image of $S$ does have the Baire property relative to $X$.

For, suppose $\varphi$ is any homeomorphism mapping $S$ onto a set $T$ contained in $X$, and let $G$ be any open set in $X$ which has a nonempty intersection with $T$. The set $G_{1}=G \cap T$ being open relative to $T$, the set $E_{1}=\varphi^{-1}\left(G_{1}\right)$ is a nonempty set open relative to $S$ and consequently $E_{1}=E \cap S$ for some set $E$ open in $X$. Since $X-K$ is the union of an everywhere dense set of intervals contained in $S$, there is an open interval $I$ contained in $E_{1}$. Hence $H=\varphi(I)$ is a nonempty open set contained in $G$ for which $H \cap(X-T)=$ $\varnothing$ is of the first category. Thus $T$ has the Baire property.

We recall that a set is always of the first category if and only if it is of the first category relative to every perfect set. The measure-theoretic analogue of such sets are the sets which are absolutely of measure zero and these sets have been shown by Marczewski to be identical, in the case of linear sets, to those sets every homeomorphic image of which is of Lebesgue measure zero. But, as we show next, the linear sets every homeomorphic image of which is of the first category in $X$ do not coincide with the linear sets which are always of the first category.

THEOREM 2. There exists a linear set every homeomorphic image of which is of the first category, but which is not always of the first category.

Proof. Following Bendixson, we determine in each interval contiguous to the Cantor set $K$ a decreasing sequence of points converging to the left endpoint of the interval and an increasing sequence of points converging to the right endpoint of the interval. Let $Q$ be the set of all these, denumerably many, sequences and let $S=A \cup Q$, where $A$ is defined in the proof of Theorem 1. The set $S$ is not always of the first category, since it is not of the first category relative to the Cantor set $K$.

To verify the fact that every homeomorphic image of $S$ is of the first category in $X$, suppose $\varphi$ is any homeomorphism mapping $S$ onto a subset of $X$. If $\varphi(A)$ were dense in some open interval then, in view of the fact that $A$ is a subset of the derived set $Q^{\prime}=K$ of $Q$, the set $\varphi(Q)$ would also be dense in the same interval. But this is impossible, since $Q$ is an isolated or discrete set and such sets are topologically invariant. Thus $\varphi(A)$ is nowhere dense in $X$ and so also is $\varphi(S)=\varphi(A) \cup \varphi(Q)$.

In the course of proving Theorem 2, we have actually established the existence of an uncountable set every homeomorphic image of which is nowhere dense, but which is not always of the first category and hence is not a scattered set. Recall that a linear set is scattered if and only if it contains no subset homeomorphic to the set of rational numbers or, equivalently, if it is 
nowhere dense relative to every perfect set. A somewhat stronger result is the following theorem.

THEOREM 3. There exists a denumerable linear set every homeomorphic image of which is nowhere dense, but which is not a scattered set.

Proof. Let $S=R \cup Q$ where $Q$ is the denumerable set defined in the proof of Theorem 2 and $R=\{1 / 3,2 / 3,1 / 9,2 / 9,7 / 9,8 / 9, \ldots\}$ is the set of all "endpoints" of the Cantor set $K$. By an argument similar to that used in proving Theorem 2, it is seen that every homeomorphic image of $S$ is nowhere dense in $X$. The set $R$ is denumerable and dense-in-itself and such a set, according to a theorem of Sierpiński [3], is homeomorphic to the set of all rational numbers. Therefore, $S$ is not a scattered set.

In view of the facts that the class $\mathcal{H}$ of sets every homeomorphic image of which has the Baire property does not coincide with the class of sets which have the Baire property in the restricted sense, that this latter class has been shown to furnish a satisfactory topological analogue for the class of absolutely measurable sets, that the class of absolutely measurable sets is identical to the class of perfectly measurable sets, and that the definition of the class $\mathcal{H}$ is analogous to the definition of perfect measurability, the question arises whether the class $\mathcal{H}$ also furnishes a satisfactory topological analogue for the class of absolutely measurable sets. This question must be answered negatively by virtue of the following facts.

(1) The class $\mathscr{H}$ of sets every homeomorphic image of which has the Baire property in $X$ does not form a $\sigma$-field.

(2) The class of sets every homeomorphic image of which is of the first category in $X$ does not form an ideal of sets.

(3) The class of sets every homeomorphic image of which is nowhere dense in $X$ does not form an ideal of sets.

In contrast to this situation, we note that both the class of sets which have the Baire property in the restricted sense and the class of absolutely measurable sets do form $\sigma$-fields, and the sets always of the first category and the sets absolutely of measure zero both form $\sigma$-ideals.

To establish the assertions (1) (3), we utilize the sets $A, Q, R$, as defined above and the set $P=R \cup\{0,1\}$. The set $K-P$ is a $\mathcal{G}_{\delta}$-set homeomorphic to the set of all irrational numbers and under such a homeomorphism the set $A$ corresponds to a totally imperfect set which does not have the Baire property relative to $X$, although every homeomorphic image of its complement $X-A$ does have the Baire property. The property (1) being thus established we easily obtain the validity of (2) by observing that the set $S=A \cup Q$ has the property that every homeomorphic image of it is of the first category in $X$, but the subset $A$ of $S$ does not have this property. Finally, the set $S=R \cup Q$ is a set every homeomorphic image of which is nowhere dense, but the subset $R$ of $S$ does not have this property. 


\section{REFERENCES}

1. M. Lavrentieff, Contribution à la théorie des ensembles homéomorphes, Fund. Math. 6 (1924), $149-160$.

2. J. C. Morgan II, On the absolute Baire property, Pacific J. Math. 78 (1978), 415-431.

3. W. Sierpinski, Sur une propriété topologique des ensembles dénombrables denses en soi, Fund. Math. 1 (1920), 11-16.

4. E. Szpilrajn (Marczewski), $O$ zbiorach $i$ funcjach bezwzglednie mierzalnych, C. R. Soc. Sci. Varsovie 30 (1937), 39-68. (An English translation of this article is available.)

Dipartaient of Mathematics, Calipornin Statb Polytbchinic Universtiy, Ponona, Caltorentu 91768 